\title{
INDICES AND NULLITIES OF YANG-MILLS FIELDS
}

\author{
HAJIME URAKAWA
}

\begin{abstract}
In this note, we give an upper estimate of index and nullity of every Yang-Mills field $R$ on every principal bundle $P$ with structure group $G$ over a Riemannian manifold $(M, g)$ with Ricci tensor Ric $\geq k g, k>0$, in terms of $\operatorname{dim}(M), \operatorname{dim}(G), k$, and $\|R\|_{\infty}$.
\end{abstract}

1. Introduction and statement of a result. Bourguignon and Lawson [B.L] obtained the second variation formula of Yang-Mills functional

$$
y \mathcal{M}(\nabla)=\frac{1}{2} \int_{M}\left\|R^{\nabla}\right\|^{2}
$$

introduced the notions of index $i(\nabla)$ and nullity $n(\nabla)$ of a Yang-Mills connection $\nabla$, and showed that there are no Yang-Mills connections $\nabla$ on $S^{n}, n \geq 5$, with the condition $i(\nabla)=0$ (cf. [B.L, Theorem 7.11]). Therefore it would be interesting to give estimates of the index $i(\nabla)$ and nullity $n(\nabla)$ of Yang-Mills connections $\nabla$.

Following [B.L], we prepare some notations.

Let $G$ be a compact Lie group, and $P$ a principal $G$-bundle over a compact Riemannian $n$-manifold $M$. For a faithful orthogonal representation $\rho$; $G \rightarrow O_{N}$, we consider a $G$-vector bundle $E=P \times{ }_{\rho} \mathbf{R}^{N}$ associated to $P$ by $\rho$. Each connection on $P$ corresponds to a $G$-connection $\nabla$ on $E$ (cf. [B.L]). We denote by $C_{E}$ the totality of $G$-connections on $E$. To each $G$-connection $\nabla$ on $E$, the curvature $R^{\nabla}$, given by

$$
R_{X, Y}^{\nabla}=\left[\nabla_{X}, \nabla_{Y}\right]-\nabla_{[X, Y]}
$$

for tangent vectors $X, Y$ on $M$, is a two form on $M$ with values in the bundle $5 o_{E}$ whose fiber $\mathfrak{s o}_{E, x}, x \in M$, consists of skew symmetric endomorphisms of the fiber $E_{x}$ of $E$. The norm of $R^{\nabla}$ at each point $x$ is given by

$$
\left\|R^{\nabla}\right\|_{x}^{2}=\sum_{i<j}\left\|R_{e_{i}, e_{j}}^{\nabla}\right\|^{2}
$$

where $\left\{e_{1}, \ldots, e_{n}\right\}$ is an orthonormal basis of the tangent space $T_{x} M$ and the inner product of the fiber $\mathfrak{s o _ { E , x }}$ is given by

$$
(A, B)=\frac{1}{2} \operatorname{trace}\left({ }^{t} A \circ B\right),
$$

for $A, B \in \mathfrak{s o}_{E, x}$. The Yang-Mills functional $Y \mathcal{M}$ on $C_{M}$ is defined as

$$
y \mathcal{M}(\nabla)=\frac{1}{2} \int_{M}\left\|R^{\nabla}\right\|^{2}
$$

Received by the editors August 19, 1985 and, in revised form, October 16, 1985.

1980 Mathematics Subject Classification (1985 Revision). Primary 53C80; Secondary 58G25. Key words and phrases. Yang-Mills field, index, nullity, eigenvalue. 
for each $\nabla \in C_{E}$. Moreover we define, for the sake of later use, the supremum norm $\left\|R^{\nabla}\right\|_{\infty}$ of $R^{\nabla}$ by

$$
\left\|R^{\nabla}\right\|_{\infty}=\sup _{x \in M}\left\|R^{\nabla}\right\|_{x}
$$

There exists a subbundle $\mathfrak{g}_{E}$ of $\mathfrak{s} o_{E}$ corresponding to a bundle $\mathfrak{g}_{P}:=P \times_{\text {Ad }} \mathfrak{g}$ through $\rho$. Let $\Omega^{p}\left(\mathfrak{g}_{E}\right), 0 \leq p \leq n$, be the space of $\mathfrak{g}_{E}$ valued $p$-forms on $M$. We get (cf. [B.L, p. 194]) an exterior differential $d^{\nabla} ; \Omega^{p}\left(\mathfrak{g}_{E}\right) \rightarrow \Omega^{p+1}\left(\mathfrak{g}_{E}\right)$ and the adjoint operator $\delta^{\nabla} ; \Omega^{p}\left(\mathfrak{g}_{E}\right) \rightarrow \Omega^{p-1}\left(\mathfrak{g}_{E}\right)$ corresponding to $\nabla \in \mathcal{C}_{E}$. It is known (cf. [B.L]) that $B=\nabla-\nabla^{\prime}$ belongs to $\Omega^{\mathbf{1}}\left(\mathfrak{g}_{E}\right)$ for each $\nabla, \nabla^{\prime} \in C_{E}$. An element $\nabla$ in $C_{E}$ is called a Yang-Mills connection if

$$
\left.\frac{d}{d t}\right|_{t=0} y \mathcal{M}\left(\nabla^{t}\right)=0
$$

for every one-parameter family of connections $\nabla^{t} \in C_{E}$ with $\nabla^{0}=\nabla$ and $d /\left.d t\right|_{t=0} \nabla^{t}$ $=B \in \Omega^{1}\left(\mathfrak{g}_{E}\right)$. The second variational formula is stated as follows.

THEOREM 1.1 (CF. [B.L, THEOREM 6.8]). For a Yang-Mills connection $\nabla=\nabla^{0} \in C_{E}$ and $B=d /\left.d t\right|_{t=0} \nabla^{t} \in \Omega^{1}\left(\mathfrak{g}_{E}\right)$ with $\delta^{\nabla} B=0$, we have

$$
\left.\frac{d^{2}}{d t^{2}}\right|_{t=0} y \mathcal{M}\left(\nabla^{t}\right)=\int_{M}\left(S^{\nabla}(B), B\right),
$$

where $S^{\nabla}$ is a differential operator on $\Omega^{1}\left(\mathfrak{g}_{E}\right)$ given by

$$
S^{\nabla}(B):=\nabla^{*} \nabla B+B \circ \operatorname{Ric}+2 \mathcal{K}^{\nabla}(B), \quad B \in \Omega^{1}\left(\mathfrak{g}_{E}\right) .
$$

Here $\nabla^{*} \nabla$ is the rough Laplacian on $\Omega^{p}\left(\mathfrak{g}_{E}\right)$ defined by

$$
\nabla^{*} \nabla \varphi=-\sum_{j=1}^{n}\left\{\nabla_{e_{j}} \nabla_{e_{j}} \varphi-\nabla_{D_{e_{j}} e_{j}} \varphi\right\}
$$

$\varphi \in \Omega^{p}\left(\mathfrak{g}_{E}\right)$, where $D$ is the Levi-Civita connection of $(M, g)$. The operator Ric; $T_{x} M \rightarrow T_{x} M$ is the Ricci transform defined by

$$
\operatorname{Ric}(X)=\sum_{j=1}^{n} R_{X, e_{j}} e_{j},
$$

where $R$ is the curvature tensor of $(M, g)$. And $B \circ \mathrm{Ric}$ is defined by $(B \circ \mathrm{Ric})(X):=$ $B(\operatorname{Ric}(X)), X \in T_{x} M$. The operator $\mathcal{K}^{\nabla} ; \Omega^{1}\left(\mathfrak{g}_{E}\right) \rightarrow \Omega^{1}\left(\mathfrak{g}_{E}\right)$ is defined by

$$
\mathcal{K}^{\nabla}(B)(X)=\sum_{j=1}^{n}\left[R_{e_{j}, X}^{\nabla}, B\left(e_{j}\right)\right] .
$$

Since $S^{\nabla}$ is an elliptic, selfadjoint operator on $\Omega^{1}\left(\mathfrak{g}_{E}\right)$, its restriction to $T^{0}:=$ $\operatorname{Ker}\left(\delta^{\nabla}\right) \subset \Omega^{1}\left(\mathfrak{g}_{E}\right)$ has eigenvalues $\lambda_{1}<\lambda_{2}<\cdots$ with associated finite-dimensional eigenspaces $E_{\lambda_{1}}, E_{\lambda_{2}}, \ldots$.

DEFINITION 1.2 (CF. [B.L , 6.10]). The index of a Yang-Mills connection $\nabla$ is the dimension $i(\nabla):=\operatorname{dim}\left(\bigoplus_{\lambda<0} E_{\lambda}\right)$, and the nullity of $\nabla$ is the dimension $n(\nabla):=\operatorname{dim}\left(E_{0}\right)$.

Now we can state our main theorem. 
THEOREM 1.3. Assume that $(M, g)$ is a compact Riemannian manifold whose Ricci tensor satisfies $\mathrm{Ric} \geq k g$ with a positive constant $k$. Then the index $i(\nabla)$ and the nullity $n(\nabla)$ of a Yang-Mills connection $\nabla$ over $M$ can be estimated as follows:

(I) If $k-2 \sqrt{2} n\left\|R^{\nabla}\right\|_{\infty}>0$, then $i(\nabla)=n(\nabla)=0$.

(II) If $k-2 \sqrt{2} n\left\|R^{\nabla}\right\|_{\infty}=0$, then $i(\nabla)=0$ and $n(\nabla) \leq \operatorname{dim}(G)$.

(III) In case $k-2 \sqrt{2} n\left\|R^{\nabla}\right\|_{\infty}<0$ :

(i) If $n \geq 3$, we have

$$
i(\nabla)+n(\nabla) \leq \operatorname{dim}(G)\left(1+\frac{1}{A}\right)^{A}\left\{1+(n-1) ! n^{n-1} A(1+A)^{n-1}\right\},
$$

where

$$
A:=2 \sqrt{2} k^{-1}\left\|R^{\nabla}\right\|_{\infty}-n^{-1}=(n k)^{-1}\left(2 \sqrt{2} n\left\|R^{\nabla}\right\|_{\infty}-k\right) .
$$

(ii) If $n=2$, we have

$$
i(\nabla)+n(\nabla) \leq \operatorname{dim}(G)\left(1+\frac{1}{B}\right)^{B}\left\{1+4 B^{2}\right\},
$$

where

$$
B:=4 \sqrt{2} k^{-1}\left\|R^{\nabla}\right\|_{\infty}-1=k^{-1}\left(4 \sqrt{2}\left\|R^{\nabla}\right\|_{\infty}-k\right) .
$$

REMARK 1.4. The function $(1+1 / x)^{x}$ in $x$ satisfies

$$
\lim _{x \rightarrow 0}\left(1+\frac{1}{x}\right)^{x}=1 \text { and }\left(1+\frac{1}{x}\right)^{x}<e \text { for all } x>0 .
$$

2. Proof of Theorem 1.3. Assume that the Ricci tensor of $(M, g)$ satisfies Ric $\geq k g$ with $k>0$. Put $P^{\nabla}(B):=B \circ$ Ric $+2 K^{\nabla}(B), B \in \Omega^{1}\left(\mathfrak{g}_{E}\right)$. Then we obtain

LEMMA 2.1. We have

$$
\left(P^{\nabla}(B), B\right)_{x} \geq\left(k-2 \sqrt{2} n\left\|R^{\nabla}\right\|_{\infty}\right)(B, B)_{x},
$$

for all $B \in \Omega^{1}\left(\mathfrak{g}_{E}\right)$. Here $(\cdot, \cdot)_{x}$ is the pointwise inner product on $\Omega^{1}\left(\mathfrak{g}_{E}\right)$ as in $\S 1$.

Proof. Since $(B \circ \operatorname{Ric}, B)_{x} \geq k(B, B)_{x}, B \in \Omega^{1}\left(\mathfrak{g}_{E}\right)$, we have only to show, for each $B \in \Omega^{1}\left(\mathfrak{g}_{E}\right)$,

$$
\left|\left(\mathcal{K}^{\nabla}(B), B\right)_{x}\right| \leq \sqrt{2} n\left\|R^{\nabla}\right\|_{\infty}(B, B)_{x} .
$$

Since $\left(K^{\nabla}(B), B\right)_{x}$ coincides, by definition, with

$$
\sum_{j=1}^{n}\left(\mathcal{K}^{\nabla}(B)\left(e_{j}\right), B\left(e_{j}\right)\right)_{x}=\sum_{j, k=1}^{n}\left(\left[R_{e_{k}, e_{j}}^{\nabla}, B\left(e_{k}\right)\right], B\left(e_{j}\right)\right)_{x},
$$

we have, using the Cauchy-Schwarz inequality,

$$
\begin{aligned}
\left|\left(K^{\nabla}(B), B\right)_{x}\right| & \leq \sum_{j, k=1}^{n}\left\|\left[R_{e_{k}, e_{j}}^{\nabla}, B\left(e_{k}\right)\right]\right\|_{x}\left\|B\left(e_{j}\right)\right\|_{x} \\
& \leq \sqrt{2} \sum_{j, k=1}^{n}\left\|R_{e_{k}, e_{j}}^{\nabla}\right\|_{x}\left\|B\left(e_{k}\right)\right\|_{x}\left\|B\left(e_{j}\right)\right\|_{x}
\end{aligned}
$$


due to the inequality in [B.L, Lemma 2.30]. Therefore, by definition of $\left\|R^{\nabla}\right\|_{\infty}$, the right-hand side is smaller than or equal to

$$
\begin{array}{r}
\sqrt{2}\left\|R^{\nabla}\right\|_{\infty} \sum_{k, j=1}^{n}\left\|B\left(e_{k}\right)\right\|_{x}\left\|B\left(e_{j}\right)\right\|_{x}=\sqrt{2}\left\|R^{\nabla}\right\|_{\infty}\left(\sum_{j=1}^{n}\left\|B\left(e_{j}\right)\right\|_{x}\right)^{2} \\
\leq \sqrt{2} n\left\|R^{\nabla}\right\|_{\infty} \sum_{j=1}^{n}\left\|B\left(e_{j}\right)\right\|_{x}^{2}=\sqrt{2} n\left\|R^{\nabla}\right\|_{\infty}\|B\|_{x}^{2}
\end{array}
$$

by the Cauchy-Schwarz inequality. Q.E.D.

Now we give an estimation of $i(\nabla)+n(\nabla)$ of a Yang-Mills connection $\nabla$ using the heat equation method due to Bérard-Gallot [B.G]. We denote the eigenvalues of $\left.S^{\nabla}\right|_{T^{0}}, S^{\nabla}$ and $\nabla^{*} \nabla$ counted with their multiplicities by $\tilde{\tilde{\lambda}}_{i}, \tilde{\lambda}_{i}$, and $\bar{\lambda}_{i}$, respectively. Then by the mini-max principle, we get, for each $i$,

$$
\tilde{\tilde{\lambda}}_{i} \geq \tilde{\lambda}_{i} \geq \bar{\lambda}_{i}+k-2 \sqrt{2} n\left\|R^{\nabla}\right\|_{\infty}
$$

Therefore for each $t>0$,

$$
\begin{aligned}
i(\nabla)+n(\nabla) & \leq \sum_{i=1}^{\infty} e^{-t \tilde{\tilde{\lambda}}_{i}} \leq \sum_{i=1}^{\infty} e^{-t \tilde{\lambda}_{i}} \\
& \leq e^{-t\left(k-2 \sqrt{2} n\left\|R^{\nabla}\right\|_{\infty}\right)} \sum_{i=1}^{\infty} e^{-t} \bar{\lambda}_{i} .
\end{aligned}
$$

Applying Kato's type inequality due to Hess, Schrader and Uhlenbrock in [H.S.U] to our case of the rough Laplacian $\nabla^{*} \nabla$ on $\Omega^{1}\left(\mathfrak{g}_{E}\right)$, we obtain, for each $t>0$,

$$
i(\nabla)+n(\nabla) \leq \operatorname{dim}(G) e^{-t\left(k-2 \sqrt{2} n\left\|R^{\nabla}\right\|_{\infty}\right)} Z_{M}(t) .
$$

Here $Z_{M}(t)$ is the trace of the heat kernel of $\partial / \partial t+\Delta_{M}$ of smooth functions on $M$, and $\Delta_{M}$ is the (positive) Laplacian of $(M, g)$. Moreover applying the inequality in [B.G, Corollary 2.2] to our Riemannian manifold $(M, g)$ with $\mathrm{Ric} \geq k g, k>0$, we get

$$
Z_{M}(t) \leq Z_{S^{n}}(k t), \quad t>0,
$$

where $Z_{S^{n}}(t)$ is the trace of the heat kernel of the standard unit sphere $\left(S^{n}\right.$, can). Therefore we obtain

$$
i(\nabla)+n(\nabla) \leq \operatorname{dim}(G) \operatorname{Inf}\left\{e^{-t\left(k-2 \sqrt{2} n\left\|R^{\nabla}\right\|_{\infty}\right)} Z_{S^{n}}(k t) ; t>0\right\} .
$$

It is known (cf. [B.G.M]) that, if $n \geq 2$,

$$
Z_{S^{n}}(t)=\sum_{k=0}^{\infty} m_{k} e^{-t k(k+n-1)},
$$

where

$$
m_{k}=\frac{(n+k-2) !}{k !(n-1) !}(n+2 k-1), \quad k=0,1,2, \ldots
$$


Then $Z_{S^{n}}(t)$ is estimated as follows:

(i) If $n \geq 3$,

$$
\begin{aligned}
Z_{S^{n}}(t) & \leq 1+\sum_{k=1}^{\infty}(n k)^{n-1} e^{-t n k} \\
& \leq 1+(n-1) ! n^{n-1} e^{-t n}\left(1-e^{-t n}\right)^{-n}
\end{aligned}
$$

(ii) If $n=2$,

$$
\begin{aligned}
Z_{S^{2}}(t) & \leq 1+2 \sum_{k=2}^{\infty} k e^{-t k} \\
& \leq 1+2 e^{-2 t}\left(2-e^{-t}\right)\left(1-e^{-t}\right)^{-2}
\end{aligned}
$$

Using these inequalities and putting $e^{t}=1+1 / A$ in (2.3) in case of (i), or $e^{t}=1+1 / B$ in (2.3) in case of (ii), respectively, we obtain the desired estimates of $i(\nabla)+n(\nabla)$ in case of (III). In case of (I) or (II), using the inequalities (2.1), (2.2) and the fact that $\lim _{t \rightarrow \infty} Z_{M}(t)=1$, we obtain the desired results. Q.E.D.

REMARK 2.2. The analogous estimates of indices and nullities for harmonic maps are obtained in $[\mathbf{U}]$.

ACKNOWLEDGMENT. The author would like to express his thanks to the referee who suggested an improvement of the first version of this paper.

\section{REFERENCES}

[B.G.M] M. Berger, P. Gauduchon and E. Mazet, Le spectre d'une variété riemannienne, Lecture Notes in Math., vol. 194, Springer-Verlag, Berlin and New York, 1971.

[B.G] P. Bérard and S. Gallot, Inégalités isopérimétriques pour l'équation de la chaleur et application a l'estimation de quelques invariants, Seminaire Goulaouic-Meyer-Schwartz, No. 15, 1983.

[B.L] J. P. Bourguignon and B. Lawson, Stability and isolation phenomena for Yang-Mills fields, Comm. Math. Phys. 79 (1981), 189-230.

[H.S.U.] H. Hess, R. Schrader and D. A. Uhlenbrock, Kato's inequality and the spectral distribution of Laplacians on compact Riemannian manifolds, J. Differential Geom. 15 (1980), 27-38.

[U] H. Urakawa, Stability of harmonic maps and eigenvalues of Laplacian (preprint).

Department of MAThematics, College of General EduCation, Tôhoku UNIVERSITY, KAWAUCHI, SENDAI, 980, JAPAN 